Research Paper

\title{
Garlic Oil Suppressed Nitrosodiethylamine-Induced Hepatocarcinoma in Rats by Inhibiting PI3K-AKT-NF-KB Pathway
}

\author{
Cui-Li Zhang, Tao Zeng, Xiu-Lan Zhao, Ke-Qin Xie ${ }^{凶}$ \\ Institute of Toxicology, School of Public Health, Shandong University, 44 Wenhua West Road, Jinan 250012, Shandong, P.R. China. \\ $\triangle$ Corresponding author: Tel.: +86 5318838 2132; Fax: +86 5318838 2129. E-mail addresses: keqinx@sdu.edu.cn.
}

(C) 2015 Ivyspring International Publisher. Reproduction is permitted for personal, noncommercial use, provided that the article is in whole, unmodified, and properly cited. See http://ivyspring.com/terms for terms and conditions.

Received: 2014.10.11; Accepted: 2015.03.03; Published: 2015.04.25

\begin{abstract}
To explore the underlying mechanisms for the protective effects of garlic oil (GO) against nitrosodiethylamine (NDEA)-induced hepatocarcinoma, 60 male Wistar rats were randomized into 4 groups $(n=15)$ : control group, NDEA group, and two GO plus NDEA groups. The rats in GO plus NDEA groups were pretreated with GO (20 or $40 \mathrm{mg} / \mathrm{kg}$ ) for 7 days. Then, all rats except those in control group were gavaged with NDEA for 20 weeks, and the rats in GO plus NDEA groups were continuously administered with GO. The results showed that GO co-treatment significantly suppressed the NDEA-induced increases of alpha fetal protein (AFP) level in serum, nuclear atypia in H\&E staining, sirius red-positive areas and proliferating cell nuclear antigen (PCNA) expression. The molecular mechanisms exploration revealed that the protein levels of phosphatidylinositol 3 kinase (PI3K)-p85, PI3K-p110, total AKT, p-AKT (Ser473) and p-AKT (Thr308) in the liver of NDEA group rats were higher than those in control group rats. In addition, NDEA treatment induced IKB degradation and NF-KB p65 phosphorylation, and up-regulated the protein levels of downstream pro-inflammatory mediators. GO co-treatment significantly reversed all the above adverse effects induced by NDEA. These results suggested that the protective effects of GO against NDEA-induced hepatocarcinoma might be associated with the suppression of PI3K- AKT-NF-KB pathway.
\end{abstract}

Key words: Garlic oil; Nitrosodiethylamine; Hepatocarcinoma; Phosphatidylinositol 3 kinase (PI3K); AKT; $\mathrm{NF}-\mathrm{KB}$

\section{Introduction}

Hepatocellular carcinoma (HCC) is a complex disease affecting thousands of people. The number of new cases of HCC is reported to be $>700,000$ per year, and more than $80 \%$ of them are detected in developing countries [1]. In China, the primary HCC is the second most common malignancy, which could result in 360,000 new cases and 350,000 deaths a year [2]. A worse scenario is that the occurrence of HCC is tended to be younger in recent decades [3]. Unfortunately, the available treatment for HCC is still disappointing $[4,5]$. Therefore, the prevention of HCC is of great importance.
$\mathrm{N}$-nitrosodiethylamine (NDEA) is one of the most important environmental carcinogens, usually existing in cheese, soybean, processed meats, alcoholic beverages, tobacco products, cosmetics and agricultural chemicals [6-8]. NDEA can induce carcinoma in all animal species, as well as in humans [9]. The carcinogenic effect of NDEA is particularly associated with the overproduction of reactive oxygen species (ROS) which could damage biomolecules such as DNA, lipids, and proteins [10, 11]. NDEA could cause the formation of large amounts of 8-hydroxy-2-deoxyguanosine $(8-\mathrm{OHdG})$ in rat liver 
even at very low dose level, which could then initate carcinogenesis [12]. Importantly, hepatocarcinogenesis induced by NDEA is an ideal animal model to investigate liver tumor formation, as it proceeds in stages similar to that of human liver cancer. Thus, NDEA-induced hepatocarcinoma animal model has been used to evaluate the chemoprotective effects of many natural compounds in a variety of studies.

The medicinal use of garlic dates back to thousands of years, and it is still one of the most popular herbal remedies in the world [13]. The mostly studied benefical effect of garlic is the anticarcinogenic activity, which has been demonstrated by a variety of epidemiological and experimental studies. However, the underlying mechanisms for the anticancer effects of garlic have not fully understood even several action modes have been proposed [14]. Garlic oil (GO), produced by steam-distillation, is a commercially available garlic product which contains several organosulfur compounds [15]. Three compounds, i.e. diallyl sulfide (DAS), diallyl disulfide (DADS), and diallyl trisulfide (DATS), are believed to be the major bioactive components of GO [13]. It has been reported that GO only accounts for about $0.1 \%$ of garlic cloves but contains the major biological components of garlic, and thus has gained great interest in recent studies. In our previous study, we have demonstrated that GO could effectively inhibit NDEA-induced hepatocarcinogenesis in male Wistar rats [16]. However, the detailed mechanism remains to be elucidated.

The phosphatidylinositol 3 kinase (PI3K)-AKT pathway is known to play important roles in numerous cellular functions such as cell growth, proliferation, differentiation, motility, survival and intracellular trafficking [17]. PI3K-AKT pathway has been reported to play critical roles in the development of a variety of tumors $[18,19]$, and also in the tumor's potential response to treatments [20]. At present, it remains unclear whether PI3K-AKT pathway plays an important role in NDEA-induced HCC. The PI3K-AKT signal pathway regulates a cascade of changes through broad target proteins including NF-kB, which has been demonstrated to be a central link between hepatic injury, fibrosis and hepatocellular carcinoma [21-23]. It has been suggested that increased reactive oxygen species (ROS) might contribute to tumorigenesis by activating NF- $\mathrm{kB}$ signal pathway [24]. However, it was still unclear whether the protective effects of GO against NDEA-induced hepatocarcinogenesis was associated with the suppression of NF-kB pathway, although our previous study has detected ROS overexpression in NDEA-induced hepatocarcinogenesis [25].

The current study was designed to evaluate the hepatoprotective effects of GO against
NDEA-induced HCC and to explore the underlying mechanisms. Male Wistar rats were pretreated with different doses of GO and then exposed to a low doses of NDEA for 20 weeks as we previously reported. We focused on PI3K-AKT-NF-KB pathway and the changes of the following pro-inflammatory factors. GO was found to almost completely suppress NDEA-activated PI3K-AKT-NF-kB pathway, which added a novel mechanism for the anticancer effects of GO.

\section{Materials and Methods}

\section{Materials}

GO was purchased from Xuchang Yuanhua Biotechnology, Inc. (Xuchang, China). The contents of DADS, DATS and DAS in GO were $31.3 \%, 29.3 \%$, $8.15 \%$, respectively. NDEA and the antibody against $\beta$-actin were obtained from Sigma-Aldrich Co. (St. Louis, MO, USA). Primary antibodies against PI3K-p85, PI3K-p110, total AKT, p-AKT (Ser473), p-AKT (Thr308), IкBa, p-IкBa (Ser32), NF-кB p65, p-NF-kB (Ser536) and proliferating cell nuclear antigen (PCNA) were obtained from Cell Signaling Technology (Beverly, MA, USA). The antibodies against cyclooxygenase 2 (COX-2), inducible nitric oxide synthase (iNOS) and vascular endothelial growth factor (VEGF) were supplied by Abcam (Cambridge, UK). Antibody against p-AKT (Tyr450), western blot detecting reagent (enhanced chemiluminescence, ECL) and polyvinylidenefluoride membrane were provided by Millipore (Bedford, MA, USA). BCA ${ }^{\mathrm{TM}}$ protein assay kits were purchased from Pierce Biotechnology (Rockford, IL, USA). Rat alpha fetal protein (AFP) ELISA kit was purchased from BlueGene Biotech (Shanghai, China).

\section{Animals and treatments}

Specific pathogen-free male Wistar rats (120-140 g) were purchased from Laboratory Animal Center of Shandong University. Rats were housed individually at $22 \pm 2^{\circ} \mathrm{C}$ with $50 \pm 10 \%$ relative humidity and a $12-\mathrm{h}$ light: 12-h dark cycle. They had free access to standard chow and tap water. After 1 week of acclimation, the rats were randomized into 4 groups $(n=15)$, i.e. control group, NDEA group, and two GO plus NDEA groups. The rats in GO plus NDEA groups were gavaged with different doses of GO (20 or $40 \mathrm{mg} / \mathrm{kg}$, dissolved in corn oil) for 7 days, while animals in other groups were treated with equal volume of corn oil. After that, all rats except those in control group orally treated with NDEA (10 mg/ $\mathrm{kg}$, 5 time/week) for 20 weeks, and the rats in GO plus NDEA groups were continuously administered with GO (4 hours ahead of NDEA treatment) until the end of the ex- 
periment. All animals were sacrificed following overnight fast at the scheduled time. The liver tissue was dissected quickly and divided into two parts. A small portion of the tissue was fixed in $4 \%$ paraformaldehyde solution, and the remaining tissue was frozen in liquid nitrogen and then stored at $-80^{\circ} \mathrm{C}$ until analysis. All procedures were approved by the Ethical Committee of School of Public Health, Shandong University, China.

\section{Serum AFP detection by ELISA kit}

The blood samples were centrifuged at $1500 \mathrm{~g}$ for 20 minutes at $4{ }^{\circ} \mathrm{C}$ to obtain serum. The serum AFP level was assayed by using a rat AFP ELISA kit. The procedure was completed strictly according to the manufacturer's instructions.

\section{Histological examination}

Paraformaldehyde-fixed liver samples were paraffin-embedded, cut into $4 \mu \mathrm{m}$ sections and stained in hematoxylin and eosin (H\&E) solutions to examine morphological changes as previously reported [16]. Sirius red staining techniques were used to assess the fibrosis of the liver using Picro Sirius Red Kit (Abcam, UK).

\section{Immunohistochemistry assay}

To detect the expression of PCNA in rat livers, the paraffin sections $(4 \mu \mathrm{m})$ were subjected to antigen retrieval and blocking of endogenous peroxidase activity, followed by incubation with PCNA monoclonal antibody (diluted at 1:200) overnight at $4^{\circ} \mathrm{C}$. After that, the sections were incubated with biotinylated anti-mouse IgG for 2 hours at room temperature. The sections in negative control group were only incubated with biotinylated anti-mouse IgG. Signal detection was conducted using 3-amino-9-ethylcarbazole (AEC) chromogen (Sigma, USA). Proliferating cells were quantified by counting PCNA-positive cells and total number of cells at five arbitrarily selected fields from each tumor at $400 \times$ magnification. Proliferation index was determined as the number of PCNA-positive cells $\times 100 /$ total number of cells [26].

\section{Western blot analysis}

The lysates of liver tissue were prepared in ice-cold RIPA buffer (50mM Tris- $\mathrm{HCl}, 150 \mathrm{mM} \mathrm{NaCl}$, $1 \%$ Triton $\mathrm{X}-100,1 \%$ sodium deoxycholate, $0.1 \%$ sodium dodecyl sulfate [SDS], $1 \mathrm{mM}$ phenylmethylsulfonyl fluoride [PMSF] and 1\% cocktail protein inhibitors). Western blot analysis was performed as we previously reported [25]. In brief, protein samples were separated by electrophoresis in $10 \%$ or $12 \%$ denatured polyacrylamide gel, transferred to a polyvinylidenefluoride membrane. The membrane was blocked with $5 \%$ nonfat milk solution, and then in- cubated with the primary antibody against PI3K-p85, PI3K-p110, total AKT, p-AKT (Ser473), p-AKT (Thr308), p-AKT (Tyr450), ІкBa, p-ІкBa (Ser32), NF-кB p65, p-NF-кB p65 (Ser536), COX-2, iNOS, or VEGF overnight at $4{ }^{\circ} \mathrm{C}$ followed by horseradish peroxidase (HRP)-conjugated anti-rabbit or anti-mouse IgG for 1 hour at room temperature. The proteins were visualized using an ECL detection reagent. To ensure equal loading, the results were normalized by $\beta$-actin.

\section{Statistical analysis}

All data were expressed as mean \pm standard deviation and analyzed by SPSS13.0. The comparison among experimental groups was conducted with one-way ANOVA, while the multiple comparisons by LSD method. $P<0.05$ or $P<0.01$ was considered statistically significant.

\section{Results}

\section{GO suppressed NDEA-induced increase of the serum AFP levels}

Alpha fetal protein (AFP) is a protein produced by the liver and yolk sac of a developing baby during pregnancy. The content of AFP declines soon after birth, and increased AFP level is accepted as a biomarker for HCC [27]. NDEA administration led to significant increase of the serum AFP level, which was obviously attenuated by GO co-treatment. Compared with the control group, the serum AFP level in NDEA group rats was increased by $65.2 \%(P<0.01)$. Compared with the NDEA group rats, the serum AFP levels were decreased by $15.8 \%$ and $26.3 \%$ in $20 \mathrm{mg} / \mathrm{kg}$ GO plus NDEA group and $40 \mathrm{mg} / \mathrm{kg}$ GO plus NDEA group, respectively $(P<0.01)$ (Figure 1$)$. The protective effect of higher dose of GO was superior to that of lower dose of GO $(P<0.05)$.

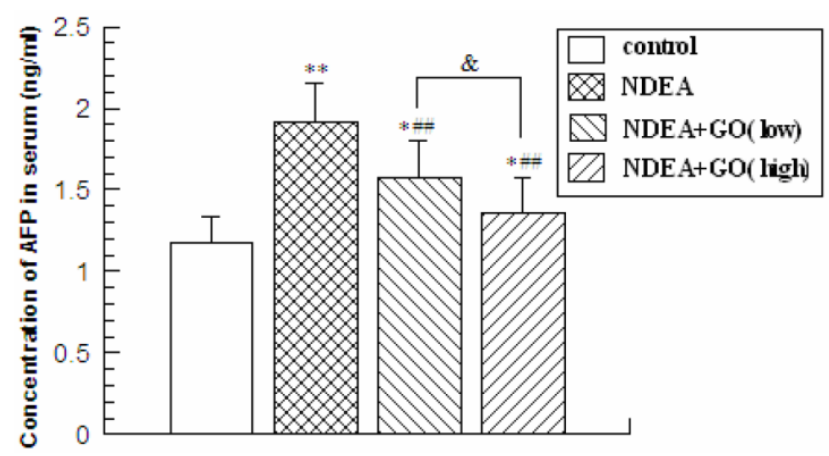

Figure 1. The levels of serum AFP $(\mathrm{ng} / \mathrm{ml})$ in rats. The serum was obtained from the blood samples by centrifugation at $1500 \mathrm{~g} \times 20 \mathrm{~min}$ for the measurement of the AFP levels with an ELISA kits. * compared with control group; $\ldots P<0.01$, compared with NDEA group; \&, $P<0.05$. 


\section{Effects of GO and NDEA on the morphological changes in rat liver}

As shown in Figure 2A, NDEA treatment led to significant loss of the hepatic architecture and the obvious nuclear atypia such as the increase of nucleus/cytoplasm ratio, disappearance of nuclear membrane, the increase of pathological karyomitosis (H\&E staining). The number of pathological karyomitosis was more than 10/10 HPF (High power field) in NDEA group. In addition, NDEA treatment resulted in the hepatocyte plate thickening and the cytoplasmic basophilia increase. GO co-treatment obviously improved hepatocyte plate arrangement and decreased the nuclear atypia. The number of pathological karyomitosis was about 5/10 HPF and less than $2 / 10 \mathrm{HPF}$ in $20 \mathrm{mg} / \mathrm{kg}$ GO plus NDEA group and $40 \mathrm{mg} / \mathrm{kg}$ GO plus NDEA group, respectively. The morphological changes of hepatocyte in GO plus NDEA groups were mainly inflammatory cell infiltration and hepatocyte edema.
The sirius red staining was used to assess the fibrosis of liver because hepatic fibrosis is a manifestation of hepatic precancerous lesion. As shown in Figure 2B, the sirius red-positive areas were markedly increased in the NDEA-treated rats, which indicating significant liver fibrosis. Interestingly, the liver sections of rats in GO plus NDEA groups showed less sirius red-positive areas.

\section{GO inhibited the increase of PCNA expression induced by NDEA in rat liver}

As shown in Figure 3A, the positive staining of PCNA occasionally appeared in the rat sections of the control group, while presented dramatical increase in NDEA group. However, GO co-treatment significantly decreased the number of PCNA positive nuclei as compared with NDEA group (Figure $3 \mathrm{~A}$ and $3 \mathrm{~B}$ ). Furthermore, the intervention effect of $40 \mathrm{mg} / \mathrm{kg}$ GO was better than that of $20 \mathrm{mg} / \mathrm{kg} \mathrm{GO}(P<0.01)$.

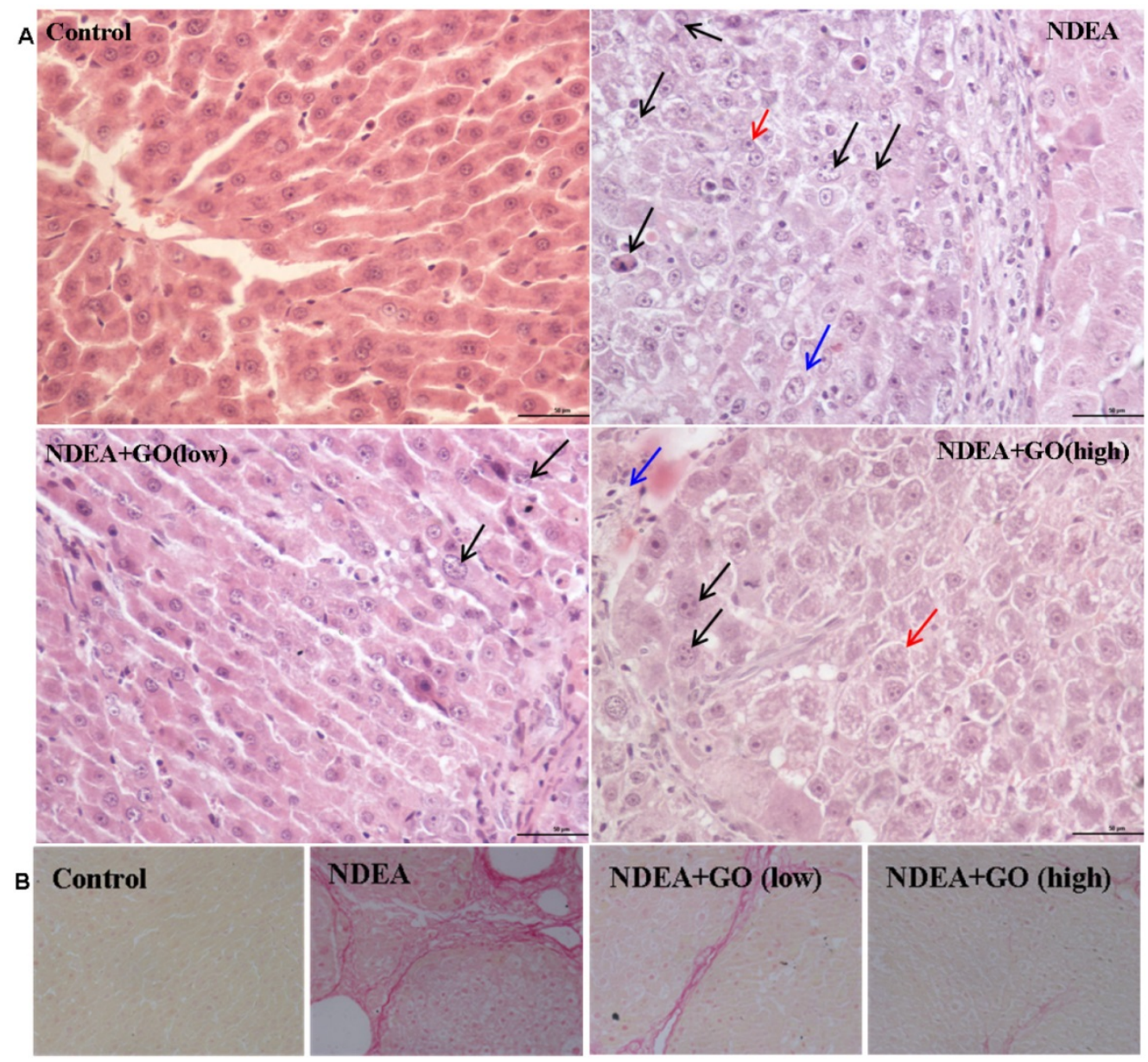

Figure 2. Effect of GO and NDEA on the morphological changes in rat livers. The histological examinations were performed followed with standard protocols. A: H\&E staining $(\times 400)$. Bar: $50 \mu \mathrm{m}$. (1) NDEA group: Black arrow points to nuclear atypia. Blue arrow points to nucleus/cytoplasm ratio increase. Red arrow points to hepatocyte plate thickening. (2) GO low dose treatment group: Black arrow points to nuclear atypia. (3) GO high dose treatment group: Black arrow points to nucleolus increase. Blue arrow points to inflammatory cell infiltration. Red arrow points to hepatocytes edema. B: Sirius red staining ( $\times 400)$. The sirius red-positive areas indicated significant liver fibrosis. 

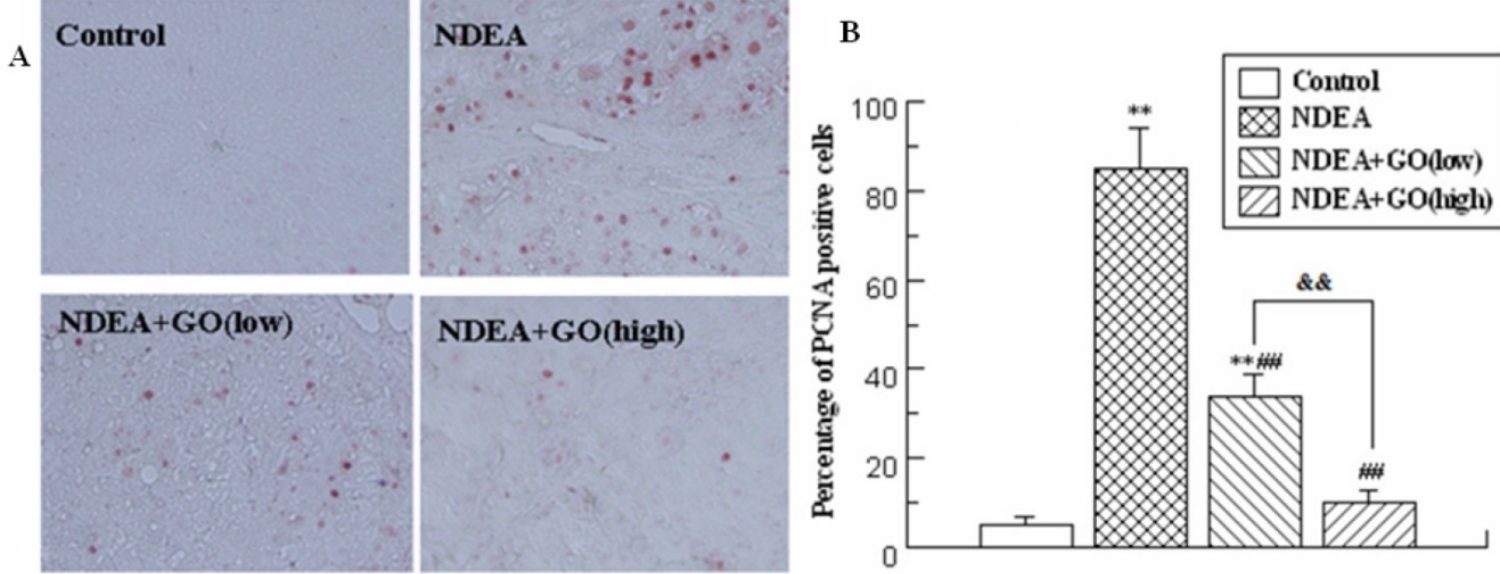

Figure 3. Effect of GO and NDEA on the PCNA expression in rat livers. A: Immunohistochemical examination of the PCNA levels ( $\times 400)$; B: Quantification of immunohistochemical sections of PCNA. **P<0.01, compared with control group; $\# P<0.01$, compared with NDEA group; \&\&, $P<0.01$.

$\mathbf{A}$

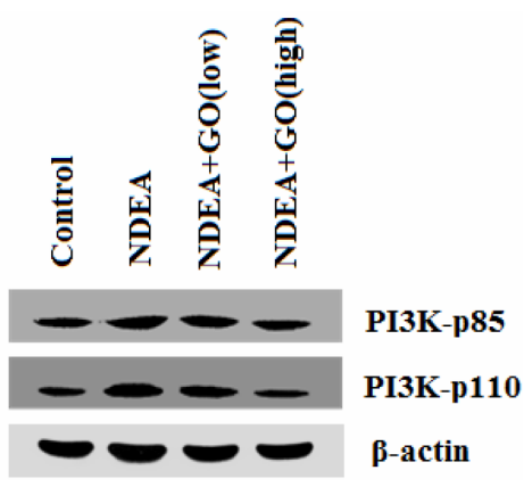

B

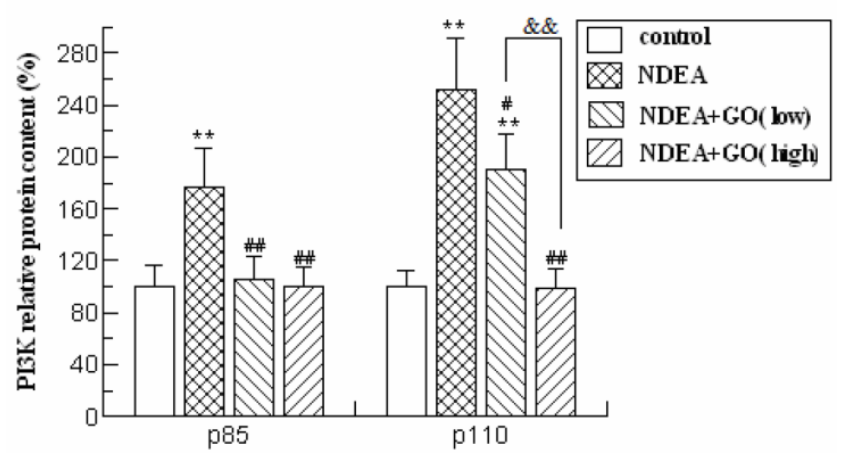

Figure 4. Effect of GO and NDEA on PI3K-p85 and PI3K-p1 10 protein contents. A: A representative immunoblot. B: Data presented the expression of $\mathrm{p} 85$ and $\mathrm{p} 110$ as percentage of control group (mean \pm SD) in triplicate. The protein levels were quantified with $\beta$-actin as an internal control. $* P<0.05, * * P<0.01$, compared with control group; $\# P<0.05, \# P<0.01$, compared with NDEA group; $\& \&, P<0.01$.

\section{GO abolished NDEA-induced activation of PI3K-AKT pathway}

To investigate whether PI3K-AKT pathway was activated by NDEA exposure and whether GO suppressed the activation of PI3K and AKT, we firstly detected the protein levels of the catalytic subunit of
PI3K (PI3K-p110) and the regulatory subunit of PI3K (PI3K-p85) by western blot. As shown in Figure 4, the protein levels of PI3K-p85 and PI3K-p110 in NDEA group rat liver were significantly increased (about 1.8-fold and 2.5-fold) compared with the control group rats $(P<0.01)$, which were significantly inhibited by GO co-treatment.

To investigate whether AKT was activated by NDEA, the protein levels of total AKT and phosphorylated AKT at three amino acid residues (Thr308, Ser473 and Tyr450) were detected. As shown in Figure 5 , the protein levels of total AKT, p-AKT (Ser473) and p-AKT (Thr308) in rat liver of NDEA group were significantly increased compared to those of control group, while the protein level of p-AKT (Tyr450) did not significantly differ among 4 groups. Compared with those of NDEA group rats, the protein levels of total AKT, p-AKT (Ser473) and p-AKT (Thr308) in GO plus NDEA groups were significantly decreased $(P<0.05)$.

\section{GO suppressed NDEA-induced IKB degrada- tion and NF-KB p65 phosphorylation}

NDEA treatment resulted in the significant decrease of IKBa protein level, while the phosphorylated IкBa (Ser32) was markedly increased (Figure 6). The protein level of phosphorylated IкBa (Ser32) in NDEA group is about 1.5 times higher than that of control group $(P<0.01)$. In addition, NDEA exposure led to a slight increase of total NF-kB p65 protein level, as well as a dramatic increase of the protein level of phosphorylated NF-kB p65 (about 28-fold, compared to that of control group). These changes were simultaneously suppressed by GO co-treatment (Figure 6, $P<0.01$, respectively). 


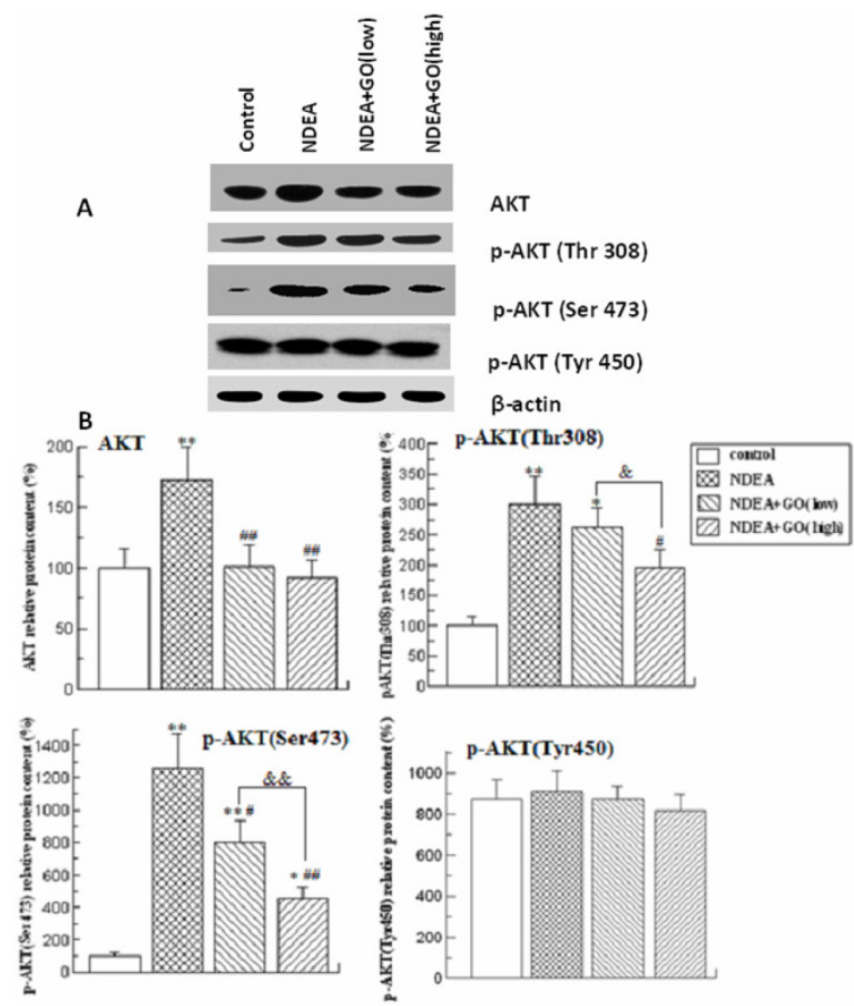

Figure 5. Effect of GO and NDEA on total AKT, p-AKT (Thr308), p-AKT (Ser473) and p-AKT (Tyr450) protein contents. A: A representative immunoblot. B: Data presented the expressions of total AKT, p-AKT (Thr 308), p-AKT (Ser473) and p-AKT (Tyr450) as percentage of control group (mean \pm SD) in triplicate. The protein levels were quantified with $\beta$-actin as an internal control. $* P<0.05$, $* * P<0.01$, compared with control group; $\# P<0.05, \# P<0.01$, compared with NDEA group; \&, $P<0.05, \& \&, P<0.01$.

\section{GO prevented the increase of COX-2, iNOS and VEGF protein levels in rat liver induced by NDEA}

As shown in Figure 7, the protein levels of COX-2, iNOS and VEGF were markedly increased $(P<0.01)$ in the liver of NDEA group rats compared to those of the control group rats. However, GO co-treatment led to dose-dependent decreases of COX-2, iNOS and VEGF protein levels $(P<0.05)$.

\section{Discussion}

The anti-cancer effects of garlic have been proposed since ancient times. Our previous studies have demonstrated that GO could significantly block NDEA-induced hepatocarcinogenesis, while the underlying mechanisms remain to be elucidated. PI3K-AKT-NF-KB pathway is believed to play important roles in the development of many tumors. In this study, we tested the changes of several important molecules in this pathway trying to elucidate the potential roles of PI3K-AKT-NF-KB pathway and the protective effects of GO against NDEA-induced hepatocarcinoma. As we previously reported, GO
A
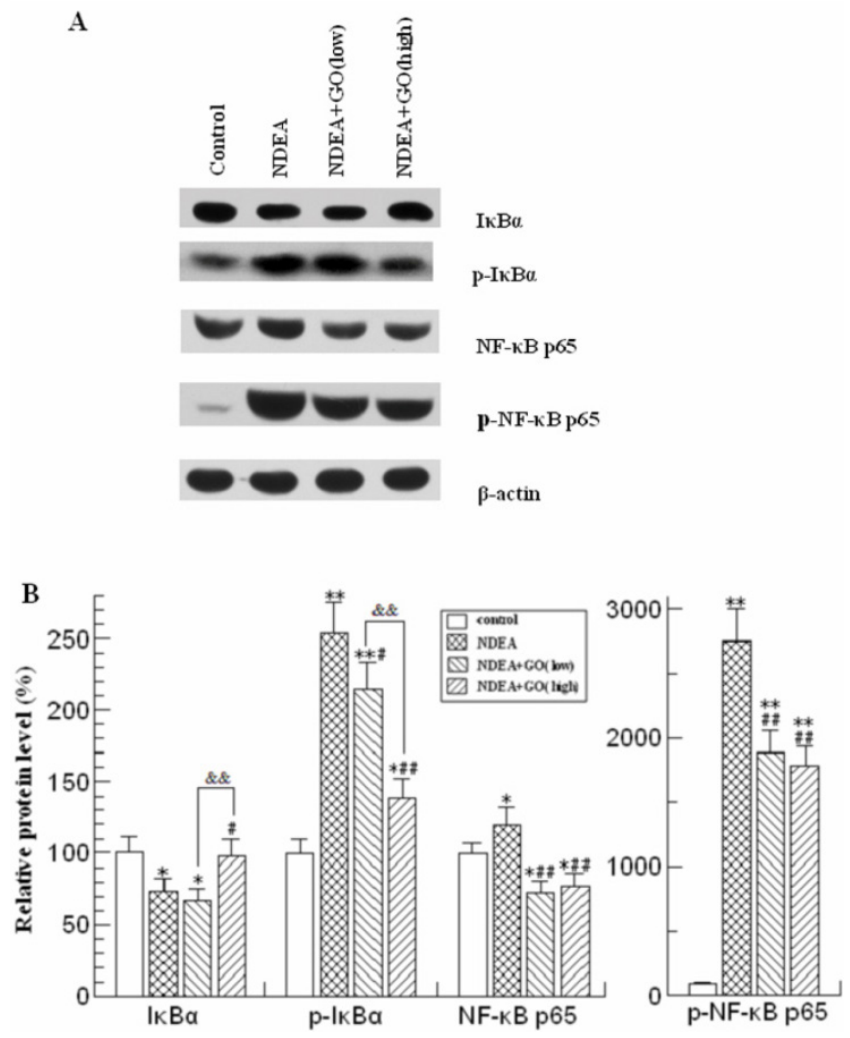

Figure 6. Effect of GO and NDEA on the protein levels of IKB, $p-I K B, N F-\kappa B$ $p 65$ and $p-N F-k B$ p65. A: A representative immunoblot. $B$ : Data presented the expressions of IKB, $\mathrm{p}-\mathrm{IKB}, \mathrm{NF}-\mathrm{kB}$ p 65 and $\mathrm{p}-\mathrm{NF}-\mathrm{kB}$ p 65 as percentage of control group (mean $\pm S D$ ) in triplicate. The protein levels were quantified with $\beta$-actin as an internal control. $* P<0.05$, $* * P<0.01$, compared with control group; $\# P<0.05, \# P<0.01$, compared with NDEA group; \&\&, $P<0.01$.

significantly inhibited the carcinogenic effects of NDEA in liver, evidenced by the decreased generation of AFP, the improvement of the morphological changes, the decrease of PCNA expression, and the molecular mechanisms exploration revealed that NDEA exposure led to the activation of the PI3K-AKT-NF-KB pathway, which were completely suppressed by GO co-treatment. Considering the key roles of PI3K-AKT-NF-KB pathway in the development of a variety of tumors, we speculated that the anticarcinogenic effects of GO against NDEA might be at least partially related to the suppression of the activation of PI3K-AKT-NF-KB pathway.

PI3Ks are a family of enzymes involved in cellular functions such as cell growth, proliferation, differentiation, motility, survival and intracellular trafficking, which in turn are involved in cancer. The PI3K family is divided into three different classes: Class I, Class II, and Class III, based on primary structure, regulation, and in vitro lipid substrate specificity[28]. The Class I PI3Ks are responsible for the production of $\mathrm{PIP}_{3}$, which could bind to the pleckstrin homology domain of AKT and phosphoinosi- 
tide-dependent protein kinase 1 (PDK1), leading to the phosphorylation and activation of AKT [29-31]. In the current study, NDEA treatment significantly increased the protein levels of the catalytic subunit (PI3K-p110) and the regulatory subunit (PI3K-p85) of PI3K. Then the high expressions of PI3K-p110 and PI3K-p85 catalyzed the production of $\mathrm{PIP}_{3}$ and resulted in the phosphorylation and activation of AKT, which could be evidenced by the increases of the total AKT, p-AKT (Ser473) and p-AKT (Thr308) protein levels. However, the expression of p-AKT (Tyr450) in NDEA group remained unchanged when compared with corresponding control value. These results strongly demonstrated PI3K-AKT pathway had been activated in the rats treated with NDEA. As expected, GO co-treatment inhibited the increases of PI3K-p85, PI3K-p110, total AKT, p-AKT (Ser473) and p-AKT (Thr308) induced by NDEA. It has been reported that the expression of phospho-AKT was correlated with a series of clinico-pathologically relevant parameters of hepatocarcinoma patients by immunohistochemical technique [32]. The activation of AKT promotes the invasion and metastasis of cancer cells. The activated AKT will further recruit and phosphorylate intracellular signaling adaptor proteins and trigger a number of signaling pathways that regulate cancer cell invasion and metastasis [33]. Thus, the inhibition of AKT phosphorylation and activation might be the critical procedures in the preventive effects of GO on NDEA-induced hepatocarcinoma.

NF- $\mathrm{kB}$, an important downstream signal molecule of PI3K-AKT pathway, is a heterodimer of transcription factor p65 and transcription factor p50 [34, 35]. NF-kB has been demonstrated to be a key inflammatory factor in tumorigenesis [36] and has been shown to be up-regulated in human hepatocarcinoma [37]. In unstimulated cells, NF- $\mathrm{kB}$ binds to I $\mathrm{kB}$, the NF- $\mathrm{kB}$ inhibitor. After IкB undergoes phosphorylation and degradation, NF- $\mathrm{kB}$ is released and activated $[23,38]$. One study showed that the inhibition of NF- $\mathrm{kB}$ activity significantly reduced the proliferation and invasion of Hep3B cell line, which demonstrated that the inhibition of NF-kB may be a potential therapeutic target for HCC [39]. Besides, it has been reported that the increase of total ІкBa but the decrease of IKBa phosphorylation is an important intervention target inhibiting tumor cells metastasis [40]. In this study, we examined the protein levels of IкBa and phosphorylated IкBa, and found that NDEA exposure led to the phosphorylation of IKBa increase, which was consistent with the increase of p-NF-kB p65 protein level (active form of NF-kB). Interestingly, GO co-treatment significantly suppressed the above effects of NDEA. Therefore, it could be speculated that GO inhibited the degradation of IkBa induced by
NDEA, and promoted NF-kB in the resting state, which might contribute to its protective effects against NDEA-induced hepatocarcinogenesis.

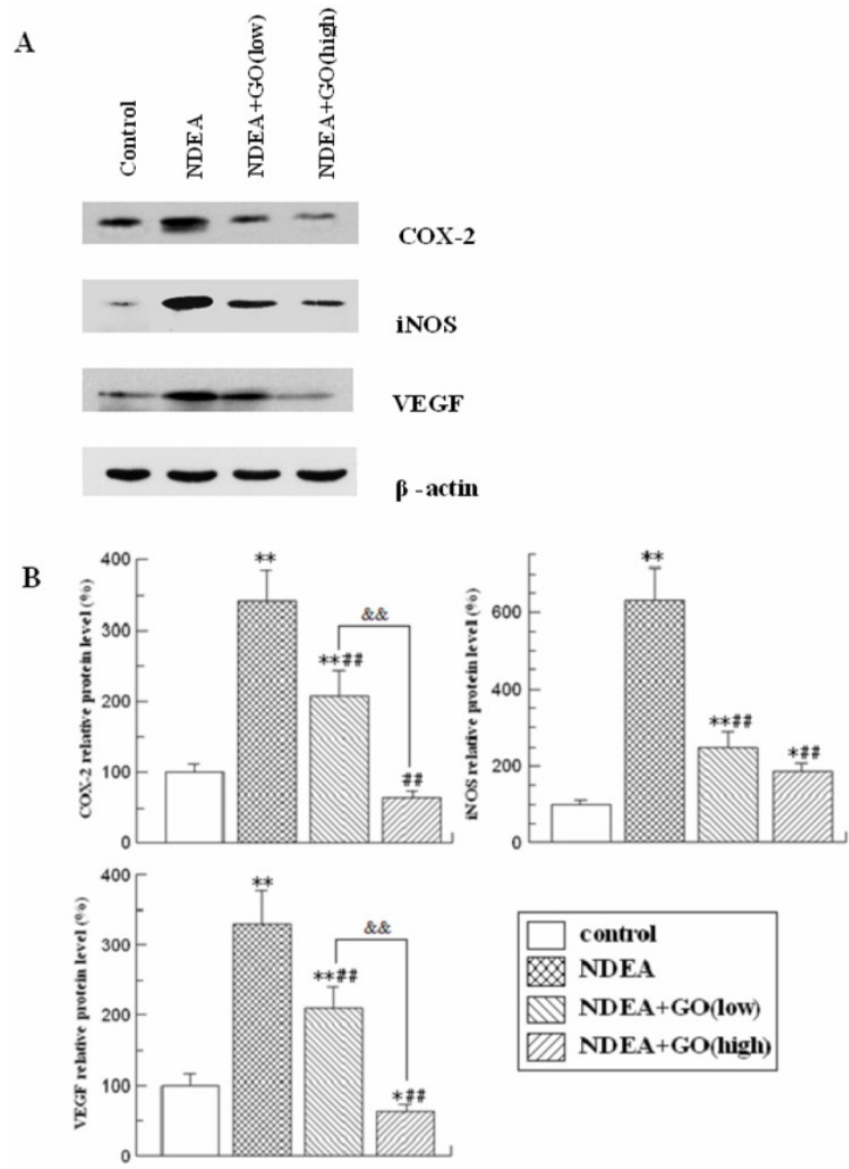

Figure 7. Effect of GO and NDEA on the protein expressions of COX-2, iNOS and VEGF. A: A representative immunoblot. B: Data presented the expressions of COX-2, iNOS and VEGF as percentage of control group (mean \pm SD) in triplicate. The protein levels were quantified with $\beta$-actin as an internal control. $* P<0.05$, $* * P<0.01$, compared with control group; $\# P<0.05, \# P<0.01$, compared with NDEA group; \&\&, $P<0.01$.

In the nucleus, NF-kB dimers bind to target DNA elements and activate the transcriptions of pro-inflammatory mediators, including COX-2, iNOS, VEGF and TNF-a, resulting in inflammation and tumorigenesis [41]. The expression of COX-2 in HCC was found to be correlated with the levels of several key molecules implicated in carcinogenesis such as iNOS and VEGF [42-44]. COX-2 is the key enzyme required for the conversion of arachidonic acid to prostaglandins. Increased expression of COX-2 has been associated with inflammatory processes and tumorigenesis, e.g., in gastrointestinal tumors, lung cancers, and gliomas [45]. Recent evidences indicated that overexpression of COX-2 and iNOS might contribute to VEGF-induced angiogenesis [43, 46]. In our study, NDEA exposure was found to lead to dramatic up-regulation of COX-2, iNOS and VEGF protein lev- 
els, which were significantly attenuated by GO co-treatment. Cherng et al. proposed that the topical application of DAS before ultraviolet B irradiation $\left(180 \mathrm{~mJ} / \mathrm{cm}^{2}\right)$ caused a delay in skin tumor formation in SKH-1 hairless mice by inhibiting NF-кB, COX-2, prostaglandin E2 (PGE2), and nitric oxide (NO) levels [47]. Shrotriya et al. showed that the inhibitory effects of DATS on 12-Otetradecanoylphorbol-13-acetate (TPA)-induced COX-2 expression by AKT inhibition may partly explain its antitumor effect on mouse skin carcinogenesis [48]. Therefore, we inferred that the inhibitory effect of GO on NDEA-induced hepatocarcinoma also involved pro-inflammatory mediators, including COX-2, iNOS and VEGF.

Some evidence indicated that ROS was an important activator for the PI3K-AKT-NF-kB pathway. For example, N-acetyl-L-cysteine (NAC), a classical antioxidant, strongly restrained the lipopolysaccharide (LPS)-induced PI3K/AKT phosphorylation and the downstream IкB kinase/ ІкB activation by reducing the ROS accumulation [49]. In addition, Pelicci et al. demonstrated that increased ROS contributed to tumorigenesis by activating NF- $\mathrm{kB}$ signal pathway in colorectal cancer [24]. In our previous study, we have indicated that GO counteracted NDEA-induced oxidative stress in rats [16]. In the current study, the increases of IкB degradation and NF-kB p65 phosphorylation induced by NDEA were significantly inhibited by GO co-treatment. Therefore, it could be speculated that the suppression of GO against NF-KB signal pathway might be related to decreased ROS.

In summary, the current study demonstrated that GO co-treatment could effectively block NDEA-induced hepatocarcinoma evidenced by the inhibition of the increases of serum AFP level, the PCNA expression, and the improvement of the hepatic histology examination. GO significantly attenuated the increases of PI3K-p110 and PI3K-p85, and AKT phosphorylation induced by NDEA. Accordingly, IкBa degradation, NF-кB p65 phosphorylation and upregulated expressions of COX-2, iNOS and VEGF were also inhibited by GO co-treatment. These results suggested that the protective effects of GO against NDEA-induced hepatocarcinoma might be associated with the suppression of PI3K-AKT-NF-кB pathway.

\section{Acknowledgements}

This work was supported by Scientific and technological cooperation project of Shandong Province [2008GG2NS02012] and National Science Foundation of Shandong Province [ZR2014HM078].

\section{Competing Interests}

The authors have declared that no competing interest exists.

\section{References}

1. Rehman MU, Buttar QM, Khawaja MI, et al. An impending cancer crisis in developing countries: are we ready for the challenge? Asian Pac J Cancer Prev. 2009; 10:719-720.

2. Chen JG, Zhang SW. Liver cancer epidemic in China: past, present and future. Semin Cancer Biol. 2011; 21:59-69.

3. Bosch FX, Ribes J, Díaz M, et al. Primary liver cancer: worldwide incidence and trends. Gastroenterology. 2004; 127:S5-S16.

4. Cheng J, Huang T, Li Y, et al. AMP-Activated Protein Kinase Suppresses the In Vitro and In Vivo Proliferation of Hepatocellular Carcinoma. PLoS One. 2014; 9.

5. Gao Q, Chen X, Duan H, et al. FXYD6: a novel therapeutic target toward hepatocellular carcinoma. Protein Cell. 2014; 10:10.

6. Subramanian P, Mirunalini S, Dakshayani KB, et al. Prevention by melatonin of hepatocarcinogenesis in rats injected with $\mathrm{N}$-nitrosodiethylamine. J Pineal Res. 2007; 43:305-312.

7. Park DH, Shin JW, Park SK, et al. Diethylnitrosamine (DEN) induces irreversible hepatocellular carcinogenesis through overexpression of G1/S-phase regulatory proteins in rat. Toxicol Lett. 2009; 191:321-326.

8. Amin A, Hamza AA, Bajbouj K, et al. Saffron: a potential candidate for a novel anticancer drug against hepatocellular carcinoma. Hepatology. 2011; 54:857-867.

9. Loeppky RN. The mechanism of bioactivation of N-nitrosodiethanolamine. Drug Metab Rev. 1999; 31:175-193.

10. Boitier E, Merad-Boudia M, Guguen-Guillouzo C, et al. Impairment of the mitochondrial respiratory chain activity in diethylnitrosamine-induced rat hepatomas: possible involvement of oxygen free radicals. Cancer Res. 1995; 55:3028-3035.

11. Yamada K, Yamamiya I, Utsumi H. In vivo detection of free radicals induced by diethylnitrosamine in rat liver tissue. Free Radic Biol Med. 2006; 40:2040-2046.

12. Nakae $\mathrm{D}$, Kobayashi $\mathrm{Y}$, Akai $\mathrm{H}$, et al. Involvement of 8-hydroxyguanine formation in the initiation of rat liver carcinogenesis by low dose levels of N-nitrosodiethylamine. Cancer Res. 1997; 57:1281-1287.

13. Zeng $\mathrm{T}$, Zhang $\mathrm{CL}$, Zhao $\mathrm{XL}$, et al. The roles of garlic on the lipid parameters: a systematic review of the literature. Crit Rev Food Sci Nutr. 2013; 53:215-230.

14. Thomson M, Ali M. Garlic [Allium sativum]: a review of its potential use as an anti-cancer agent. Curr Cancer Drug Targets. 2003; 3:67-81.

15. Zeng T, Zhang CL, Song FY, et al. Garlic oil alleviated ethanol-induced fat accumulation via modulation of SREBP-1, PPAR-alpha, and CYP2E1. Food Chem Toxicol. 2012; 50:485-491.

16. Zhang $\mathrm{CL}$, Zeng $\mathrm{T}$, Zhao $\mathrm{XL}$, et al. Protective effects of garlic oil on hepatocarcinoma induced by $\mathrm{N}$-nitrosodiethylamine in rats. Int J Biol Sci. 2012; 8:363-374.

17. Bader AG, Kang S, Zhao L, et al. Oncogenic PI3K deregulates transcription and translation. Nat Rev Cancer. 2005; 5:921-929.

18. Kawauchi K, Ogasawara T, Yasuyama M, et al. Regulation and importance of the PI3K/Akt/mTOR signaling pathway in hematologic malignancies. Anti-Cancer Agents in Medicinal Chemistry (Formerly Current Medicinal Chemistry-Anti-Cancer Agents). 2009; 9:1024-1038.

19. Furukawa T. Molecular targeting therapy for pancreatic cancer: current knowledge and perspectives from bench to bedside. Journal of gastroenterology. 2008; 43:905-911.

20. Fresno Vara JA, Casado E, de Castro J, et al. PI3K/Akt signalling pathway and cancer. Cancer Treat Rev. 2004; 30:193-204.

21. Chen J. Multiple signal pathways in obesity-associated cancer. Obes Rev. 2011; 12:1063-1070.

22. Chen J, Crawford R, Xiao Y. Vertical inhibition of the PI3K/Akt/mTOR pathway for the treatment of osteoarthritis. J Cell Biochem. 2013; 114:245-249.

23. Luedde T, Schwabe RF. NF-kappaB in the liver--linking injury, fibrosis and hepatocellular carcinoma. Nat Rev Gastroenterol Hepatol. 2011; 8:108-118.

24. Pelicci PG, Dalton P, Giorgio M. The other face of ROS: a driver of stem cell expansion in colorectal cancer. Cell Stem Cell. 2013; 12:635-636.

25. Zhang $\mathrm{CL}$, Zeng $\mathrm{T}$, Zhao $\mathrm{XL}$, et al. Garlic oil attenuated nitrosodiethylamine-induced hepatocarcinogenesis by modulating the metabolic activation and detoxification enzymes. Int J Biol Sci. 2013; 9:237-245.

26. Ito T, Shiraki K, Sugimoto K, et al. Survivin promotes cell proliferation in human hepatocellular carcinoma. Hepatology. 2000; 31:1080-1085.

27. Androutsopoulos G, Gkogkos P, Decavalas G. Mid-trimester maternal serum HCG and alpha fetal protein levels: clinical significance and prediction of adverse pregnancy outcome. Int J Endocrinol Metab. 2013; 11:102-106.

28. Leevers SJ, Vanhaesebroeck B, Waterfield MD. Signalling through phosphoinositide 3-kinases: the lipids take centre stage. Current opinion in cell biology. 1999; 11:219-225.

29. Martelli AM, Evangelisti C, Chiarini F, et al. The phosphatidylinositol 3-kinase/Akt/mTOR signaling network as a therapeutic target in acute myelogenous leukemia patients. Oncotarget. 2010; 1:89-103. 
30. Ogita S, Lorusso P. Targeting phosphatidylinositol 3 kinase (PI3K)-Akt beyond rapalogs. Target Oncol. 2011; 6:103-117.

31. Zeng T, Zhang CL, Song FY, et al. PI3K/Akt pathway activation was involved in acute ethanol-induced fatty liver in mice. Toxicology. 2012; 296:56-66.

32. Schmitz KJ, Wohlschlaeger J, Lang H, et al. Activation of the ERK and AKT signalling pathway predicts poor prognosis in hepatocellular carcinoma and ERK activation in cancer tissue is associated with hepatitis $C$ virus infection. J Hepatol. 2008; 48:83-90.

33. Yan W, Fu Y, Tian D, et al. PI3 kinase/Akt signaling mediates epithelial-mesenchymal transition in hypoxic hepatocellular carcinoma cells. Biochem Biophys Res Commun. 2009; 382:631-636.

34. Cantley LC. The phosphoinositide 3-kinase pathway. Science. 2002; 296:1655-1657.

35. Maddika S, Ande SR, Wiechec E, et al. Akt-mediated phosphorylation of CDK2 regulates its dual role in cell cycle progression and apoptosis. J Cell Sci. 2008; 121:979-988.

36. Dolcet X, Llobet D, Pallares J, et al. NF-kB in development and progression of human cancer. Virchows Arch. 2005; 446:475-482.

37. Tai DI, Tsai SL, Chang YH, et al. Constitutive activation of nuclear factor $\mathrm{KB}$ in hepatocellular carcinoma. Cancer. 2000; 89:2274-2281.

38. Hou $\mathrm{CH}$, Tang $\mathrm{CH}, \mathrm{Hsu} \mathrm{CJ}$, et al. $\mathrm{CCN} 4$ induces IL-6 production through alphavbeta5 receptor, PI3K, Akt, and NF-kappaB singling pathway in human synovial fibroblasts. Arthritis Res Ther. 2013; 15:R19.

39. Wu JM, Sheng $H$, Saxena $R$, et al. NF-kappaB inhibition in human hepatocellular carcinoma and its potential as adjunct to sorafenib based therapy. Cancer Lett. 2009; 278:145-155.

40. Guo K, Kang NX, Li Y, et al. Regulation of HSP27 on NF-kappaB pathway activation may be involved in metastatic hepatocellular carcinoma cells apoptosis. BMC Cancer. 2009; 9:100.

41. Pan MH, Ho CT. Chemopreventive effects of natural dietary compounds on cancer development. Chem Soc Rev. 2008; 37:2558-2574.

42. Koga H, Sakisaka S, Ohishi M, et al. Expression of cyclooxygenase-2 in human hepatocellular carcinoma: relevance to tumor dedifferentiation. Hepatology. 1999; 29:688-696.

43. Rahman MA, Kohno H, Nagasue N. COX-2 - a target for preventing hepatic carcinoma? Expert Opin Ther Targets. 2002; 6:483-490.

44. Jahan S, Khaliq S, Ijaz B, et al. Role of HCV Core gene of genotype 1a and 3a and host gene Cox-2 in HCV-induced pathogenesis. Virol J. 2011; 8:155.

45. Hara A, Okayasu I. Cyclooxygenase-2 and inducible nitric oxide synthase expression in human astrocytic gliomas: correlation with angiogenesis and prognostic significance. Acta Neuropathol. 2004; 108:43-48.

46. Kanayama S, Yamada Y, Kawaguchi R, et al. Hepatocyte growth factor induces anoikis resistance by up-regulation of cyclooxygenase-2 expression in uterine endometrial cancer cells. Oncol Rep. 2008; 19:117-122.

47. Cherng JM, Tsai KD, Perng DS, et al. Diallyl sulfide protects against ultraviolet B-induced skin cancers in SKH-1 hairless mouse: analysis of early molecular events in carcinogenesis. Photodermatol Photoimmunol Photomed. 2011; 27:138-146.

48. Shrotriya S, Kundu JK, Na HK, et al. Diallyl trisulfide inhibits phorbol ester-induced tumor promotion, activation of AP-1, and expression of COX-2 in mouse skin by blocking JNK and Akt signaling. Cancer Res. 2010; 70:1932-1940

49. Qi S, Xin Y, Guo $Y$, et al. Ampelopsin reduces endotoxic inflammation via repressing ROS-mediated activation of PI3K/Akt/NF-kappaB signaling pathways. Int Immunopharmacol. 2012; 12:278-287. 